ARTICLE

\title{
Primary cell wall inspired micro containers as a step towards a synthetic plant cell
}

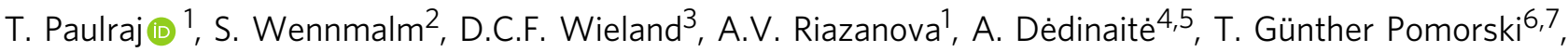 \\ M. Cárdenas ${ }^{8} \&$ A.J. Svagan ${ }^{1 凶}$
}

The structural integrity of living plant cells heavily relies on the plant cell wall containing a nanofibrous cellulose skeleton. Hence, if synthetic plant cells consist of such a cell wall, they would allow for manipulation into more complex synthetic plant structures. Herein, we have overcome the fundamental difficulties associated with assembling lipid vesicles with cellulosic nanofibers (CNFs). We prepare plantosomes with an outer shell of CNF and pectin, and beneath this, a thin layer of lipids (oleic acid and phospholipids) that surrounds a water core. By exploiting the phase behavior of the lipids, regulated by $\mathrm{pH}$ and $\mathrm{Mg}^{2+}$ ions, we form vesicle-crowded interiors that change the outer dimension of the plantosomes, mimicking the expansion in real plant cells during, e.g., growth. The internal pressure enables growth of lipid tubules through the plantosome cell wall, which paves the way to the development of hierarchical plant structures and advanced synthetic plant cell mimics.

\footnotetext{
${ }^{1}$ KTH Royal Institute of Technology, Department of Fibre and Polymer Technology, Teknikringen 56, 10044 Stockholm, Sweden. ${ }^{2}$ KTH Royal Institute of Technology, SciLifeLab, Department of Applied Physics, Biophysics, Tomtebodavägen 23a, 17165 Solna, Sweden. ${ }^{3}$ Helmholtz-Zentrum Geesthacht: Centre for Materials and Costal Research, Institute of Materials Research, Max-Planck-Straße 1, 21502 Geesthacht, Germany. ${ }^{4}$ KTH Royal Institute of Technology, Deptartment of Chemistry, Division of Surface and Corrosion Science, Drottning Kristinas väg 51, 10044 Stockholm, Sweden. ${ }^{5}$ RISE Research Institutes of Sweden, Division of Bioscience and Materials, 11486 Stockholm, Sweden. ${ }^{6}$ Ruhr University Bochum, Faculty of Chemistry and Biochemistry, Department of Molecular Biochemistry, Universitätsstraße 150, 44780 Bochum, Germany. ${ }^{7}$ University of Copenhagen, Department for Plant and Environmental Sciences, Thorvaldsensvej 40, 1871 Frederiksberg C, Denmark. ${ }^{8}$ Malmö University, Biofilm - Research Center for Biointerfaces and Department of Biomedical Science, 20506 Malmö, Sweden. ${ }^{凶}$ email: svagan@kth.se
} 
T he field of synthetic biology has widened our understanding of modern animal cells, and provided inspiration and innovative ideas for material chemistry ${ }^{1-4}$. Several attempts have been made to construct synthetic animal cells ${ }^{5-7}$. However, examples of synthetic plant cells, with both cell wall and plasma membrane mimics, have to our knowledge not been reported. This is surprising, since plant cells have well-defined structures (plasmodesmata) that serve as communication bridges across individual cells ${ }^{8-10}$, and a synthetic plant cell could therefore serve as a simple model to understand intracellular communication. A major challenge when constructing synthetic plant cells is to prepare a continuous cellulose microfibril layer on top of biomimetic plasma membranes ${ }^{11}$. In natural plant cells, a cell wall surrounds the plasma membrane ${ }^{12}$. In parenchyma cells, the wall is a thin primary cell wall that encompasses a nanofibrous cellulosic network, pectin, hemicellulose, and minor fractions of structural proteins ${ }^{13}$. The so-called cellulosic microfibrils, typically having a width of $\sim 4 \mathrm{~nm}$ and several micrometers in length, are essential for the mechanical and structural integrity of the plant cell wall ${ }^{13}$. Cellulosic microfibrils can be extracted from plants in the form of cellulosic nanofibers $(\mathrm{CNFs})^{14}$. However, due to their considerable length and semicrystalline nature, it has not been possible to assemble CNFs on top of vesicles; lipid vesicles typically have diameters below the micrometer range, while CNFs consist of stiff crystalline segments $(\sim 300 \mathrm{~nm}$ in wood) and only allow coating formation on top of spherical structures above $\sim 600 \mathrm{~nm}$ in diameter ${ }^{15}$. Natural plant cells are $10-100 \mu \mathrm{m}$ in size ${ }^{12}$, and thus to mimic plant cells, micrometersized vesicles are necessary. Even though reconstitution of polymer and polysaccharides on giant unilamellar phospholipid vesicles (GUVs) has been demonstrated in the past, to the authors' knowledge, there are no studies to date that report GUVs coated with a continuous and dense layer of CNFs. This is because GUVs are quite fragile. CNFs, on the other hand, which form a viscous suspension in water (even at low concentrations), are highly entangled and difficult to process. In other words, CNF suspensions present other challenges compared to dissolved polymer solutions. Recently, more robust GUVs were successfully prepared, by using an outer stabilizing layer of block copolymers to first encapsulate several small vesicles in water-in-oil droplets, followed by the fusion of vesicles into one single GUV inside each water droplet ${ }^{16}$. Unfortunately, such a protocol cannot be used with natural CNFs (where CNFs take the role of the block copolymer), as CNFs can only be dispersed in water. Another strategy is to exploit the unique colloidal and physicochemical properties of nanocellulose at oil/water interfaces, which can be used to self-assemble a dense CNF layer at such interfaces ${ }^{15,17}$. In this study, we use precisely these properties to obtain a continuous layer of CNFs via a modified production protocol.

Herein, the primary plant cell wall polysaccharides nanocellulose and pectin are combined with oleic acid (OA), oleate, and structural plant phospholipids to generate plant-cellinspired microcapsules, which we call plantosomes. OA has previously been used in the assembly of models of primitive cells, so-called protocells ${ }^{18,19}$. OA and oleate show rich phase behavior in aqueous media ${ }^{20-22}$, which can be utilized together with phospholipids, for an alternative fabrication route of plant cell mimics. Similar to the turgor pressure mechanism in real plant cells, the phase behavior of the OA/oleate-rich interior of plantosomes can be utilized to expand the microcapsules. Moreover, by tuning the formation conditions, the plantosome interior can be filled with a crowded lipid-based milieu that also extends through the polysaccharide capsule wall in the form of lipid tubular structures. The present study represents an important step toward fabrication of advanced synthetic plant cells, and studies of such synthetic cells in physiologically relevant settings might improve our understanding of the evolution of plant cells.

\section{Results}

Formation of $\mathrm{CNF} /$ pectin microcapsules with $\mathrm{OA} /$ oleate cores. First, we studied the self-assembly of OA/oleate and the polysaccharides to understand how the phase behavior of OA could be exploited to make artificial plant cells. OA forms an oil-inwater emulsion at low $\mathrm{pH}(<7)$, but cubic, lamellar, and micellar phases upon increasing the $\mathrm{pH}$ to, respectively, $7.5,8-9$, or even higher $^{20}$. In the present study, cationic CNFs, extracted from wood pulp, were used in the fabrication of plant cell mimics (Fig. 1a). A $288 \mathrm{mM}$ OA solution in chloroform was emulsified in the presence of an aqueous CNF suspension (0.059 wt\%), using a 1:1 volume ratio (Fig. 1b). The nanofibers accumulated at the oil/ water interface and stabilized the emulsion (Supplementary Figs. $1-3)^{15}$. In a further step, sugar beet pectin was adsorbed on top of the CNF layer (Supplementary Figs. 4 and 5, Supplementary Note 1), further providing stability and allowing microcapsule with OA/oleate cores to evolve. Both $\mathrm{CNF}$ and pectin were pivotal for stability during the microcapsule formation (Supplementary Figs. 1-4, Supplementary Method 1, Supplementary Note 1). The final microcapsules were formed by evaporating the chloroform, followed by adjusting the $\mathrm{pH}$ of the microcapsule suspension to 2 and then 6.5 (details in Supplementary Fig. 3, Supplementary Method 2, Supplementary Note 2). A schematic representation of the final microcapsule is shown in Fig. 1c. Prior to evaporation of chloroform, the CNF/ pectin-stabilized oil droplets contained both chloroform and OA, and had a diameter of $39 \pm 15 \mu \mathrm{m}$ (Fig. 1d). In addition, the interior of the oil-phase occasionally contained water droplets (arrow in Fig. 1e). The final microcapsules, on the other hand, were much smaller, $27 \pm 11 \mu \mathrm{m}$ (Fig. 1d, f), which corresponds to a significant volume decrease of $67 \mathrm{vol} \%$ and shrinking of the outer $\mathrm{CNF} /$ pectin wall area with $52 \%$, on average. The large volume shrinkage, and the $\mathrm{pH}$ (6.5) of the suspension, suggests a microcapsule interior presenting high OA content in its protonated form ${ }^{20}$ and some occasional water droplets.

To identify the interior oil and water parts, the microcapsules were exposed to dyes that labeled the hydrophobic lipid core regions (rhodamine 6G, Rh-6G) ${ }^{23}$ and the aqueous regions (sulforhodamine 101, SR-101) ${ }^{23}$ of the microcapsule interior. These dyes confirmed that the microcapsule interior consisted mainly of lipid (Fig. 2a, b, stained with Rh-6G), with a minor fraction of water droplets (Fig. 2c, d, SR-101). Polarized optical microscopy (POM, Fig. 2e) revealed Maltese crosses at the outer rim of the microcapsules, indicative for concentrically organized (lamellar) lipids.

To reveal the presence of the encasing $\mathrm{CNF} /$ pectin wall, the lipids were removed from the microcapsule interior (Supplementary Movie 1) and the remaining microcapsule walls were stained with a beta-glucan-binding dye (calcofluor-white stain; Fig. 2f). The lipid core removal was achieved by increasing the $\mathrm{pH}$, which led to the lipid solubilization into vesicles and then micelles, which could escape through the microcapsule wall. In the process, the interior volume expanded with a concomitant microcapsule radius increase, signifying a large extensibility of the encasing $\mathrm{CNF} /$ pectin wall. The radius and volume of the microcapsules in Supplementary Movie 1 increased with ca. $41 \%$ and $180 \mathrm{vol} \%$ on average, respectively, during the expansion, which is in the same order as the observed shrinkage during microcapsule preparation (Fig. 1d). The increase in the outer capsule wall area was $98 \%$ on average. Occasionally microcapsules also burst during the rapid expansion (the arrows in Fig. $2 \mathrm{f}$ point to such burst cavities). 

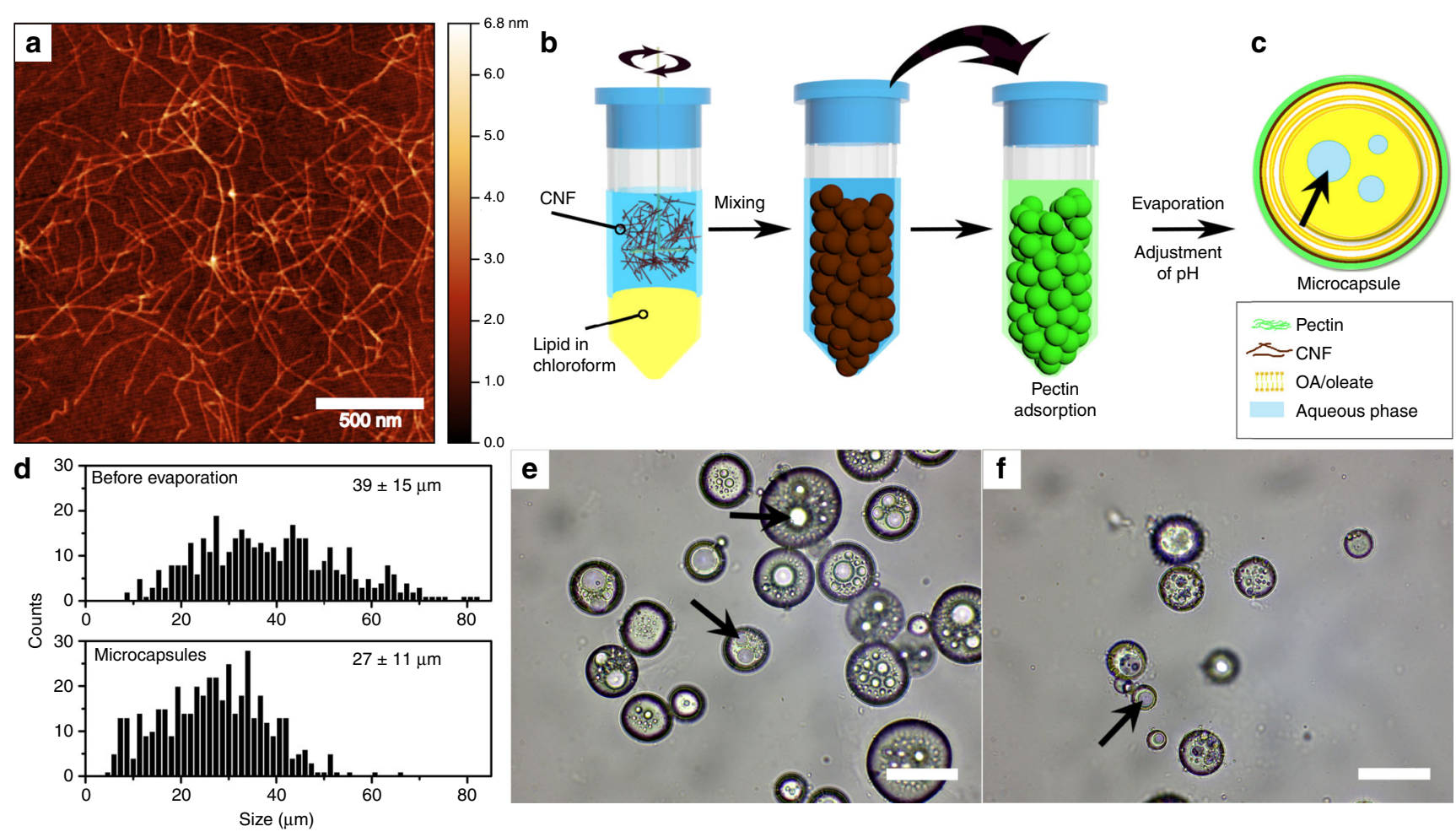

Fig. 1 Polysaccharide assembly on the surface of lipid droplets. a Representative AFM image of the cationic CNFs (derived from three experiments). b Schematic representation of the preparation of CNF/pectin-stabilized microcapsules with OA/oleate cores. c Proposed organization within the obtained microcapsule, including the organization of the OA/oleate beneath the outer CNF/pectin shell. In $\mathbf{b}$ and $\mathbf{c}$ : water-blue, lipid-yellow, pectin-green, and CNF-brown. The size distribution $\mathbf{d}$ and corresponding representative bright field images of CNF/pectin-stabilized oil droplets prior to chloroform evaporation e and for microcapsules $\mathbf{f}$. Data in $\mathbf{d}$ were collected from four experiments, and histograms includes $n=402 \mathrm{CNF} /$ pectin-stabilized oil droplets before chloroform evaporation and $n=447$ microcapsules. The average diameters and s.d. are reported. Arrows in $\mathbf{c}, \mathbf{e}$, and $\mathbf{f}$ point to encapsulated water droplets. Height bar: $0-6.8 \mathrm{~nm}$ a. Scale bars: $500 \mathrm{~nm}$ a and $50 \mu \mathrm{m}$ e, f.

Formation of plantosomes. The microcapsules in Figs. 1 and 2 contained mostly lipid in their cores. Living parenchyma cells, on the other hand, contain a water-based cytoplasm enclosed by the plasma membrane, and $\sim 1 \mathrm{wt} \%$ of lipid (hydrated state) ${ }^{24}$. To include a higher fraction of water and less lipids in the interior of the microcapsules, a small amount of phospholipids $(0.22 \mathrm{~mol} \%$ with respect to the total lipid amount) was also dissolved in the chloroform solution that was used in the production protocol. In this way, a population of microcapsules, consisting of $\mathrm{CNF} /$ pectin shells with very thin lipid layers beneath the shell and large water droplets in the interior, was attained (Fig. 3). We call them plantosomes. A mixture (1:5 mol ratio) of 1-palmitoyl-2-oleoyl$s n$-glycero-3-phosphoethanolamine (POPE) and 1-palmitoyl-2oleoyl-sn-glycero-3-phosphocholine (POPC) was used in the selfassembly process. These phospholipids are naturally found in the plasma membrane of plant cells and plasmodesmata ${ }^{9}$, representing $68-80 \%$ of the structural phospholipids ${ }^{25}$. Only a small amount of phospholipid was necessary $(0.3 \mathrm{mM}$ of phospholipid in the chloroform phase) to achieve the spontaneous selfassembly into plantosomes. The final microcapsule suspension was, still, a mixture of capsules with varying sizes of water-filled cavities (plantosomes, black arrows), and in some cases, the water cavity was missing (microcapsules, white arrows Fig. 3a, b). The diameter of the plantosomes prior to and after chloroform evaporation was $32 \pm 9 \mu \mathrm{m}$ and $20 \pm 5 \mu \mathrm{m}$, respectively (histograms in Fig. 3). The decrease in the average diameter was both due to chloroform evaporation and that larger plantosomes burst during the evaporation.

After chloroform evaporation, the $\mathrm{pH}$ of the capsule suspension was around $5.8-5.9$, which means that the OA was mainly present in its protonated form within the lipid layer ${ }^{20-22}$. The lipids were found in one or a couple of concentric rings in the periphery of the plantosomes (Fig. 3c, f). The presence of the large water compartments in the interior in the plantosomes was further verified by exposing them to the permeable water-soluble dye SR-101 (Fig. 3d). In Fig. 3e, f, the plantosomes and microcapsule also contained a rhodamine-labeled phospholipid (1,2-dioleoyl-sn-glycero-3-phosphoethanolamine- $N$-(lissamine rhodamine B sulfonyl) Rh-DOPE. A schematic representation of a plantosome is shown in Fig. 3g.

Confocal laser scanning microscopy (CLSM) studies of calcofluor-white stained plantosomes (Fig. 4a, b) provided information about the organization of the CNFs in the outer encasing shell. To elucidate details of the nanoscale structure of the $\mathrm{CNF} /$ pectin shell, we performed transmission electron microscope (TEM) and scanning electron microscope (SEM) studies of the remaining shell after lipid removal (Fig. $4 \mathrm{c}-\mathrm{e}$, Supplementary Fig. 6, Supplementary Note 3). During lipid removal, the plantosomes and microcapsules expanded, and the shells were stretched, as described previously. A shell that burst during lipid release was selected for TEM imaging (Fig. 4c-e), a technique that allowed better visualization of CNFs. Highmagnification images of different parts of the remaining shell in Fig. $4 \mathrm{c}$ are given in Fig. 4d, e. These images revealed a dense structure consisting of a network of slender nanofibrous cellulose in a pectin matrix, which demonstrated successful self-assembly of CNFs and pectin into a dense shell. This shell represents a simple model of the primary cell wall in real plant cells, which consists of a network of hemicellulose-crosslinked cellulose microfibrils embedded in a pectin matrix ${ }^{13,26}$. Nano-sized pores, 

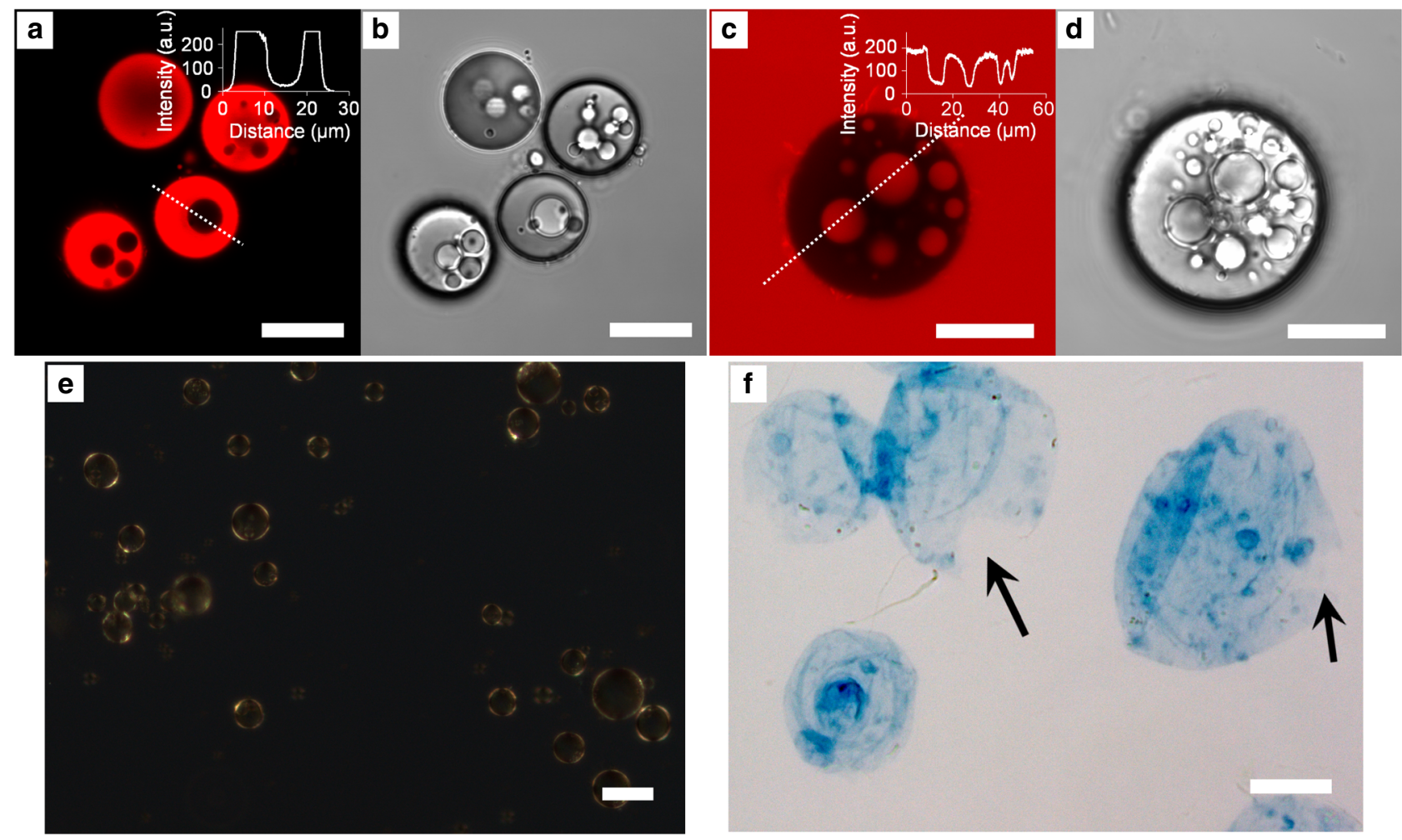

Fig. 2 Microscopy images of microcapsules with predominately lipid in the interior. CLSM images of $\mathbf{a}$, $\mathbf{b}$ microcapsules exposed to rhodamine $6 \mathrm{G}$ (Rh6G, $0.01 \mathrm{mg} \mathrm{mL}^{-1}$ ) and $\mathbf{c}$, d sulforhodamine 101 (SR-101, $0.5 \mathrm{mg} \mathrm{mL}^{-1}$ ). Insets show intensity line profiles obtained from the marked lines. $\mathbf{a}, \mathbf{c}$ and $\mathbf{b}$, $\mathbf{d}$ are fluorescence and transmission images, respectively. e The OA/oleate lipids were organized concentrically in the periphery (below the CNF/pectin capsule wall) of the microcapsules, observed as Maltese crosses in POM. In f light microscopy image of empty and collapsed microcapsule walls (i.e., devoid of $\mathrm{OA}$ /oleate cores). The capsules were emptied by increasing the $\mathrm{pH}$, see Supplementary Movie 1. The capsule walls were stained blue with calcofluor-white stain. The arrows point to burst cavities. All images are representative of three experiments. Scale bars: $20 \mu \mathrm{m}$ a-d and $\mathbf{f}$, and $50 \mu \mathrm{m}$ e.

on average $18 \pm 12 \mathrm{~nm}(n=120)$ in the dry state, were also observed in the present shell structures (best observed in the SEM images in Supplementary Fig. 6a, b) after lipid removal. Occasionally larger pores, that were several of tens of nanometers in diameter, also could be observed (Supplementary Fig. 6b). These pores are larger than for the primary cell wall in living plant cells, which consists of pores ranging from 3.5 to $5.2 \mathrm{~nm}$ (in the wet state $)^{27}$. However, it is unclear if the pores were formed during self-assembly or were a consequence of lipid removal.

In Fig. 4f, a plantosome is presented prior to expansion, where the lipid layers are still intact. We observed that plantosomes were partially permeable to small fluorescein isothiocyanate (FITC)-dextran molecules $\left(\bar{M}_{w}=4 \mathrm{kDa}\right)$ : a fraction of FITC-dextran could enter the space between the concentric lipid rings but no FITC-labeled dextran was observed in the interior water core of plantosomes. Thus, FITC-labeled dextrans with a hydrodynamic diameter of $\sim 3 \mathrm{~nm}$, could permeate though the encasing $\mathrm{CNF} /$ pectin shell but not the lipid barrier.

Expansion and formation of lipid tubular structures. Plant cells are normally under turgor pressure, which tightly presses the plasma membrane against the cell wall ${ }^{12}$. The cell wall, on the other hand, preserves the plant cell and protects it from bursting. At the same time, plant cells are able to allow tubular membrane structures to cross their cell wall and act as a communication bridge across cells. The turgor pressure is controlled by the vacuoles, which are fluid-filled compartments that are able to expand the plant cells via osmotic uptake of water. Such cell enlargement is critical during, e.g., cell growth.
We therefore tested whether it is possible to expand also our plantosomes. As our plantosomes have no vacuoles, we aimed to create a crowded lipid milieu in the interior. We allowed the interior OA/oleate/POPC/POPE lipids to self-assemble spontaneously into vesicles by gradually raising the $\mathrm{pH}$ from 5.8 to 8.6 in the presence of $0.2 \mathrm{M}$ ammonium acetate, which is a solute that is highly permeable through vesicle membranes ${ }^{20,22,28}$. Under these conditions, a pure OA/oleate/POPC/POPE mixture in 0.2 $\mathrm{M}$ ammonium acetate self-assembles into vesicles (control experiment in Supplementary Fig. 7 and Supplementary Note 4). The role of the ammonium acetate was to enable faster diffusion of buffer solutes and water into the interior of the plantosomes ${ }^{28}$, which was critical for plantosome expansion. In situ CLSM monitoring of plantosome expansion, including the proposed expansion mechanism, is presented in Fig. 5a-h (Supplementary Movie 2, transmission images in Supplementary Fig. 8). When the $\mathrm{pH}$ increased from 8.0 to 8.3 , the interior of the plantosome was filled with vesicles (Fig. 5d). The largest expansion in plantosome size was observed in the last $\mathrm{pH}$ step ${ }^{22}$. The present uptake of water during expansion was not driven by the same osmosis mechanism as in plants: the concentration of buffer solutes (except for lipids) is adjusted quickly between the interior and exterior of the plantosomes at all time points during the $\mathrm{pH}$ increase due to the presence of ammonium acetate (see Supplementary Movie 2). Water uptake was achieved when the $\mathrm{OA}$ (in the interior of the plantosomes) converted to oleate with $\mathrm{pH}$, and the oleate/OA/phospholipids self-assembled into vesicles (at $\mathrm{pH}>8$ ), as illustrated in Fig. 5g. The plantosome (Fig. 5a-f) increased $29 \%$ in radius that gives a surface area enlargement of $66 \%$, which is somewhat larger than the values obtained for a 

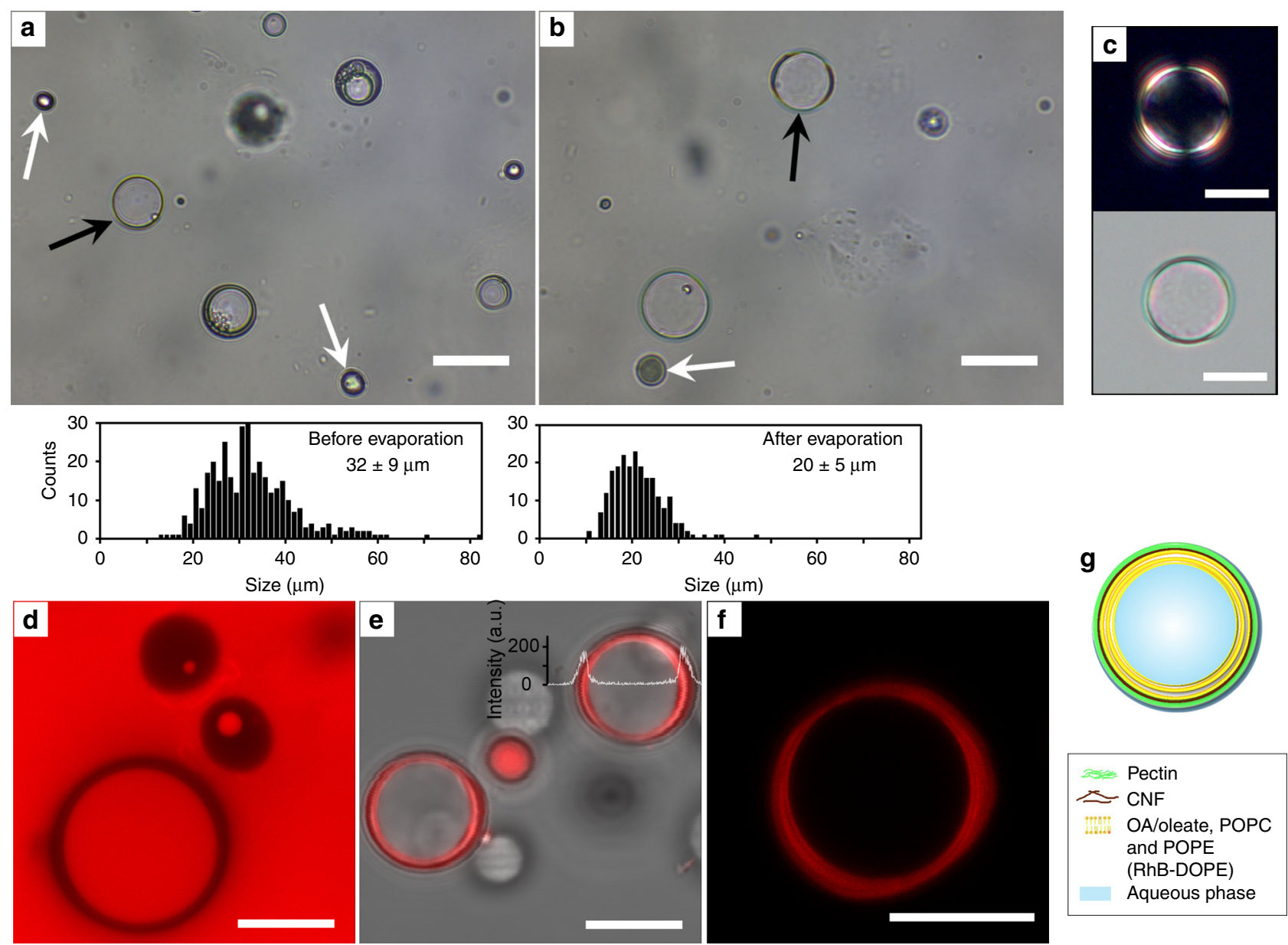

Fig. 3 Plantosomes-microcapsules with thin interior lipid layers and large water-filled cavities. a Bright field image prior to chloroform evaporation. A mix of CNF/pectin-stabilized chloroform/lipid droplets devoid of water droplets or with varying sizes of water droplets, some of which were very large and filled out a large volume of the inner core (plantosomes). The lipid phase consisted of OA, POPE, and POPC. b After chloroform evaporation, the final population consisted of capsules with a similar composition as in a. Black and white arrows point to plantosomes and microcapsules devoid of water in the interior, respectively. Histograms: the size distribution of plantosomes prior $(n=356)$ and after chloroform evaporation ( $n=218$ ). Data derived from four experiments and images in $\mathbf{a}$ and $\mathbf{b}$ are representative images in these experiments. The average diameters and s.d. are reported $\mathbf{c}$ POM (bright field) image showing the organization of lipids in a plantosome. d CLSM images of plantosome and microcapsules showing the interior water parts stained with SR-101 (0.5 mg mL $\left.{ }^{-1}\right)$. e Combined fluorescence-transmission image of plantosomes and microcapsules containing Rh-DOPE (red) in the lipid phase. A

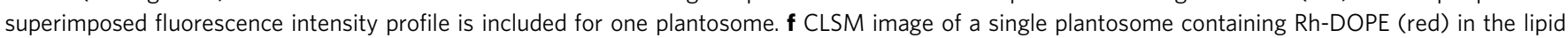
phase. The lipids are organized in a couple of concentric rings in the periphery. Images in c-f are representative of three experiments. $\mathbf{g}$ Schematic representation of the cross-section of a plantosome. Scale bars: $20 \mu \mathrm{m}$ a, b, and $10 \mu \mathrm{m} \mathbf{c}-\mathbf{f}$.

larger population of plantosomes $(n=9)$ : $13 \pm 9 \%$ in radius increase and area increase of $28 \pm 22 \%$. In Fig. 5f, the same plantosome is shown after $1 \mathrm{~h}$ at $\mathrm{pH} 8.6$ and demonstrate that the plantosome withstands the expansion over extended time periods. In Fig. 5i, j, the expansion of microcapsules (devoid of water cores), along with one plantosome, is presented. Such microcapsules $(n=4)$ were consistently enlarged to a much higher degree: in the given example, the average increase in radius was $68 \pm 8 \%$ with an increase in the surface area of $184 \pm 26 \%$. The values obtained from a larger population of microcapsules $(n=$ 22) were: $66 \pm 12 \%$ and $177 \pm 39 \%$ increase in radius and surface area, respectively (Fig. 5k, l). The larger expansion power is attributed to the high content of lipid in the interior; note that the capsule wall of microcapsules (with lipid only in the interior) also experience a large shrinkage during chloroform evaporation (Fig. 1d). In the latter case, the capsule wall demonstrated a remarkable extensibility. Previous studies showed that highly plasticized $\mathrm{CNF} /$ polysaccharide film can only be elongated in the order of $20 \%$ (at $20-40 \mathrm{wt} \% \mathrm{CNF}$ ) until it breaks ${ }^{14}$. Assuming only thinning (no necking) of the film, the area increase would be $20 \%$ for such a film, which is much lower than the observed area increase for the present microcapsule wall. Therefore, we hypothesize that the large expansion of the capsule surface cannot only be due to nanofiber pullout and capsule wall thinning, but is also enabled due to stretching a crumpled capsule wall surface. A crumpled/buckled surface area has previously been observed and reported for nanocellulose-based particles subjected to significant shrinkage during fabrication ${ }^{29,30}$. The structure arises at some point during the shrinking process, when the CNFs transit into a kinetically arrested state ${ }^{10,11}$. When this occurs, the surface buckles and a crumpled capsule wall structure, with folds on the submicron length scale, is obtained ${ }^{30}$. The exact morphology of the crumpled structure will depend on the shrinkage conditions (examples of crumpled capsules, after chloroform evaporation, are included in Supplementary Fig. 3a and 5, Supplementary Movie 1) ${ }^{10,11}$.

In all cases, the expansion resulted in a highly crowded interior lipid milieu (illustrated in Fig. $5 \mathrm{~g}$ ). The present vesicles were, 


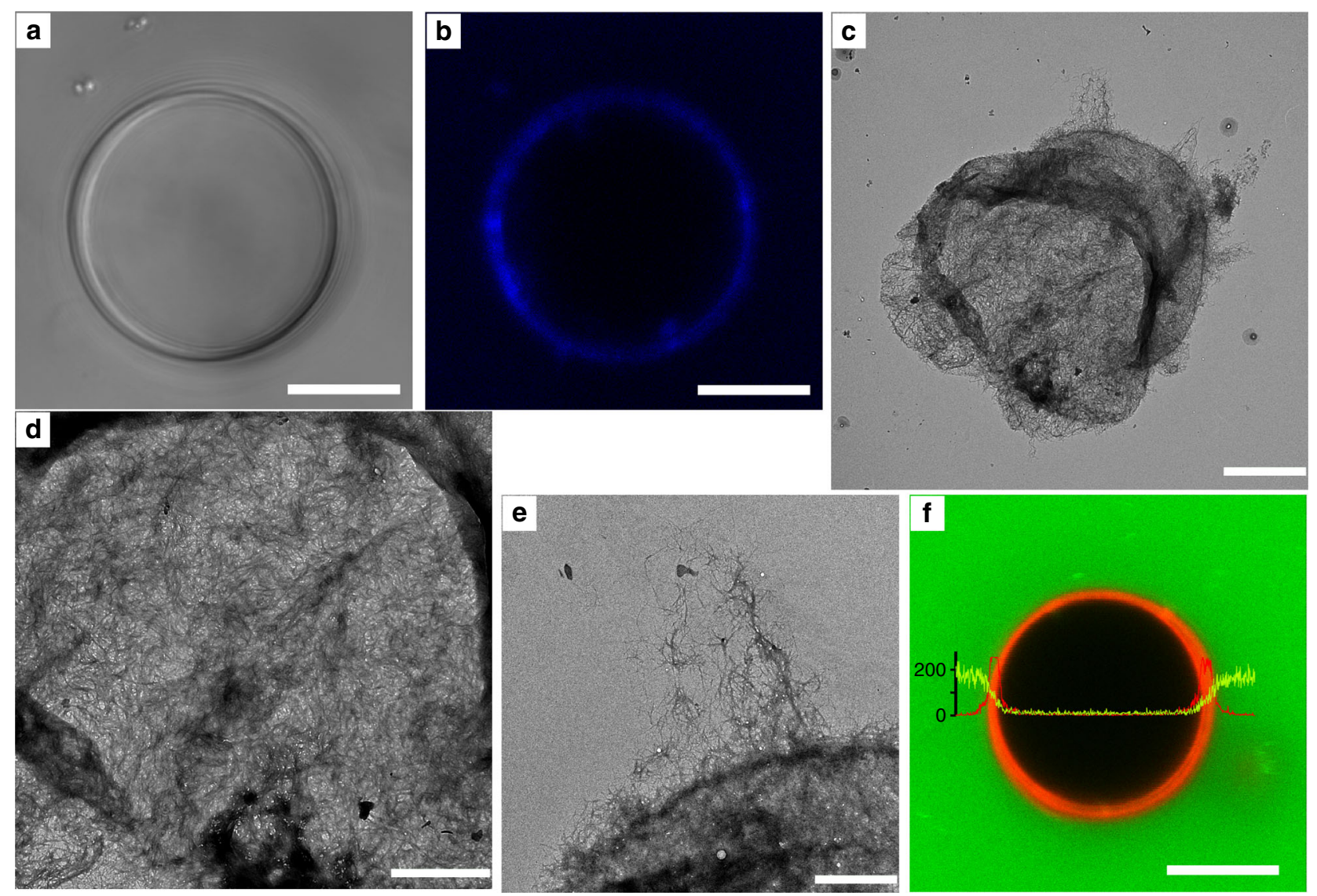

Fig. 4 The nanostructure of the encasing CNF/pectin wall. CLSM transmission a and fluorescence $\mathbf{b}$ images of a plantosome stained with calcofluor-white (blue). TEM micrographs c-e of the remaining (dry) pectin/CNF shell after removal of lipids (OA, POPC, and POPE) from the interior of plantosomes/ microcapsules. A burst cavity was created during the lipid removal. Images in $\mathbf{d}$ and $\mathbf{e}$ are high-resolution images of the shell in $\mathbf{c}$. In $\mathbf{f}$, fluorescence intensity profile for a plantosome exposed to FITC-dextran $\left(\bar{M}_{w}=4 \mathrm{kDa}, 1 \mathrm{mg} \mathrm{mL}-1\right.$, green). The lipid phase contained Rh-DOPE (red). Images a-b and c-f are representative of two and three repeated experiments, respectively. Scale bars: $10 \mu \mathrm{m} \mathbf{a}, \mathbf{b}, 5 \mu \mathrm{m} \mathbf{c}, 2 \mu \mathrm{m} \mathbf{d}, 1 \mu \mathrm{m} \mathbf{e}$, and $10 \mu \mathrm{m} \mathbf{f}$.

however, too small to be observed with CLSM (CLSM images of larger vesicles, formed by fusing these small vesicles, are shown in the next section). After expansion, all expanded structures appeared similar, irrespective of the starting structure, i.e., a plantosome or a lipid-filled microcapsules (Fig. 5i, j). Therefore, we refer to all of them as expanded plantosomes. All the formed lipid compartments of the expanded plantosomes were highly permeable to $4 \mathrm{kDa}$ FITC-dextran (Supplementary Fig. 9, Supplementary Note 5), due to the presence of $0.2 \mathrm{M}$ ammonium acetate that is known to enhance the permeability of vesicle membranes ${ }^{28}$. The absence of Maltese crosses in the interior of expanded plantosomes (Supplementary Fig. 10, Supplementary Note 6) signified that the formed lamellar structures were thin and below the detection limit of an ordinary POM. Interestingly, the strong capsule wall, CNF, and pectin, did not burst (in most cases), taking on a similar role to that of the plant cell wall in real plant cells, although pores were present in the expanded wall (Fig. 4c-e, Supplementary Fig. 6), through which the lipids could escape. The primary cell wall in parenchyma cells still withstands high turgor pressures during the growth of the plant cells, despite being in a highly hydrated state, which is primarily due to the skeletal CNF network in the cell wall ${ }^{13}$.

To release the pressure in some of the most overcrowded expanded plantosomes, tubular protrusions appeared and extended from the plantosome surfaces (Fig. 6a, faintly observed in Fig. 5j, Supplementary Movie 3, Supplementary Fig. 11, Supplementary Note 7). These protrusions remained attached to the expanded plantosome surface throughout the duration of the experiment. All of the plantosomes and lipid-filled microcapsules expanded during the $\mathrm{pH}$ increase (to 8.6), however, in some cases the $\mathrm{CNF} /$ pectin walls burst during expansion $(10 \%$ of an expanded plantosome population of $n=116$ ), which led to a halting of further expansion (Supplementary Fig.11b). For these, tubular protrusions were not observed (Supplementary Fig. 11b). But when the CNF/pectin wall did not burst, almost all of the expanded plantosomes exhibited tubular protrusions (we estimate that to be $\sim 80 \%, n=102$ ), but a larger numbers of protrusions were observed from the surface of an expanded plantosomes that had completely been filled with lipid prior to expansion (e.g., a previous microcapsule devoid of a water core). OA/oleate vesicles have previously been reported in literature to grow as thread-like/ tubular vesicles that are predisposed to divide ${ }^{28,31}$. These were created by feeding multilamellar $\mathrm{OA} /$ oleate vesicles with additional micelles to the exterior of the vesicles, and as the additional lipids were incorporated much faster into the outermost lipid membrane layer compared to the inner, the outermost membrane grew by forming protrusions ${ }^{28}$. An additional factor in the formation process was volume conservation between the lipid membranes in the multilamellar OA/oleate vesicle structure, due to the slow permeability of buffer solutes into the space between lipid membranes. However, in the presence of $0.2 \mathrm{M}$ ammonium acetate, the permeability was increased and the same group observed that the outermost membrane layer of a multilamellar $\mathrm{OA} /$ oleate vesicle expanded as a sphere in the presence of supplementary exterior micelles ${ }^{28}$. Herein, we observe thread-like structures, in the presence of $0.2 \mathrm{M}$ ammonium acetate. 


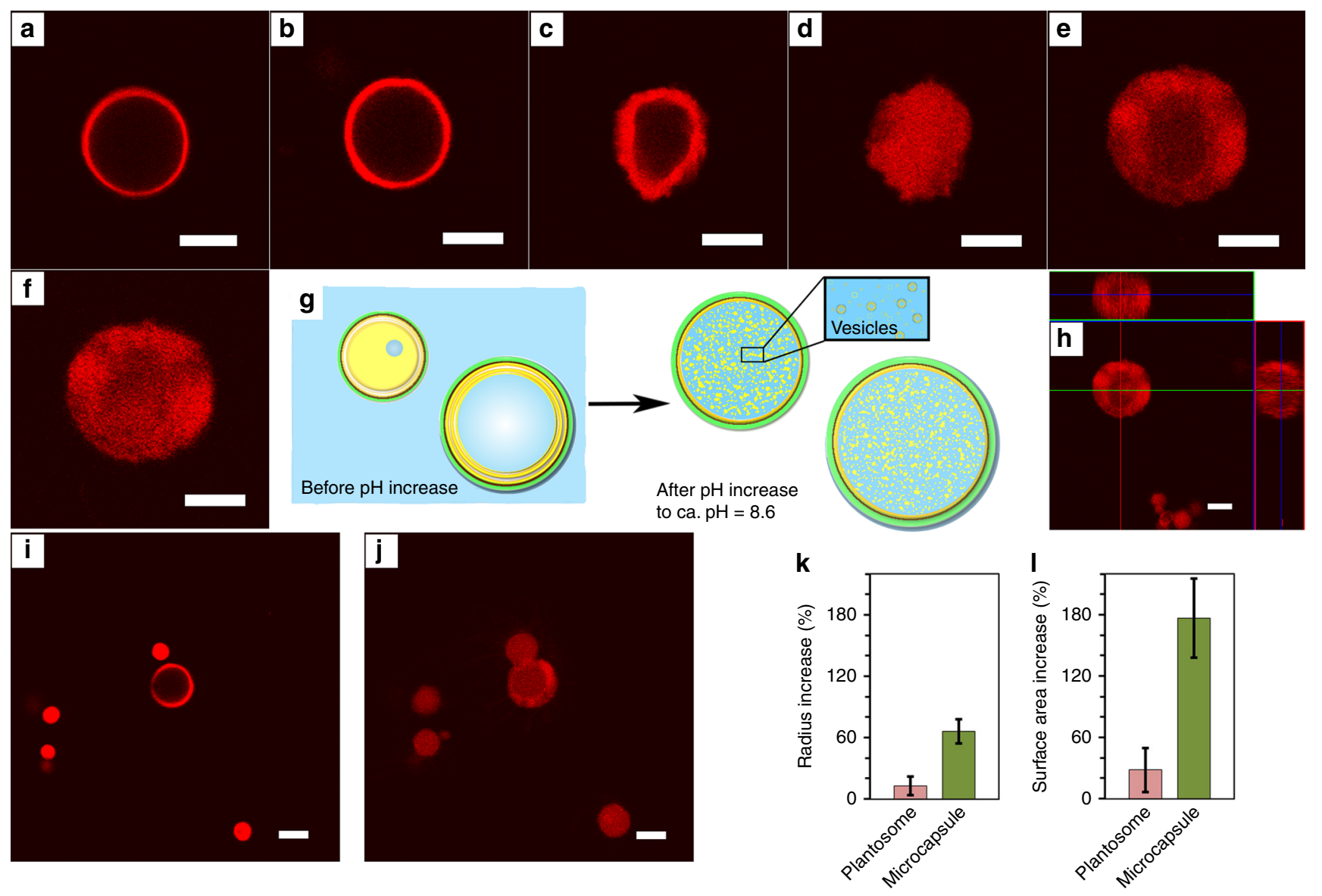

Fig. 5 Formation of vesicles inside plantosomes. CLSM images of plantosome in a $100 \mathrm{mM} \mathrm{NaCl}$ solution, $\mathbf{b}$ transferred into $0.19 \mathrm{M}$ ammonium acetate solution (with $100 \mathrm{mM} \mathrm{NaCl}$ ) at pH 6.5. Increasing the $\mathrm{pH}$ in a step-wise manner $\mathbf{c}$ to $8.0 \mathbf{d}, 8.3$, and finally e to 8.6. After the final pH increase, an expanded plantosome is created. In $\mathbf{f}$, after $1 \mathrm{~h}$ at $\mathrm{pH}$ 8.6. $\mathbf{g}$ Proposed mechanism for the formation of vesicles in the interior. $\mathbf{h}$ Orthogonal view of the expanded plantosome in $\mathbf{e}$. In $\mathbf{c}-\mathbf{h}$, the medium contained $0.2 \mathrm{M}$ ammonium acetate, $100 \mathrm{mM} \mathrm{NaCl}$. The lipid phase contained OA/oleate, POPE, POPC, and Rh-DOPE (red). The images for the experiment in a-f are representative of seven repeated experiments. In $\mathbf{i}$ and $\mathbf{j}$, the same type of experiment repeated for a plantosome and microcapsules (without water cavities). The $\mathrm{pH}$ is $6.5 \mathbf{i}$ and $8.6 \mathbf{j}$. Images in $\mathbf{i}-\mathbf{j}$ are representative of five experiments. The average increase in radius $\mathbf{k}$ and surface area $\mathbf{I}$ for a population of microcapsules ( $n=22$ microcapsules, obtained from five experiments) and plantosomes ( $n=9$ plantosomes, obtained from nine experiments). Data are presented as mean \pm s.d. Scale bars: $10 \mu \mathrm{m}$. Source data underlying $\mathbf{k}$, I are provided as a Source Data file.

Moreover, extra micelles were not added to the exterior in the present experiments, and thus the source for additional lipids could not be other than the interior of the microcapsules/ plantosomes. During expansion, the net pressure is exerted in the radial direction, and the lipid molecules will move from the interior toward the outer boundaries of the microcapsule/ plantosome. We hypothesize that our lipid tubular structures are a consequence of the strongly restraining cage-like $\mathrm{CNF} /$ pectin wall, which limits the indefinite expansion and only allows the lipid to escape through holes in the $\mathrm{CNF} /$ pectin wall (see pores in the shell of an expanded plantosomes in Supplementary Fig. 6). To further prove this, we mixed the same lipid composition with an ammonium acetate solution, but in the absence of the CNF and pectin. The resulting vesicles were only spherical in shape (control experiment shown in Supplementary Fig. 7). Studies on artificial lipid membranes (GUVs) based on POPC and Rh-DOPE, that contained a crowded and viscous (protein) interior, have shown to deform in a similar manner as reported herein, i.e., via tubing deformations extending from their surfaces. This occurred, however, when the GUVs were placed in a hypertonic solution ${ }^{32}$. The excess membrane lipids (after shrinking) formed the tubular deformations and the initial viscosity inside the liposomes was the only determinant of the type of membrane deformation (tubular or bud deformations). We envision a similar mechanism for the present expanded plantosomes, which contain a crowded interior with excess lipids and a stiff $\mathrm{CNF} /$ pectin wall that imposes a constraining counter pressure during plantosome expansion, simulating that of the osmotic pressure of a hypertonic solution in the mentioned study $^{32}$. However, to elucidate the exact formation steps for the present tubular structures, further investigations are needed. The present observation, however, implies that fatty-acid-based membranes with a small fraction of phospholipids have the ability to form tubular structures that extend beyond the boundaries of a rigid $\mathrm{CNF} /$ pectin cell wall, which has not been shown before. Pure fatty-acid-based membranes have been considered to be the earliest forms of cell membranes ${ }^{19,33}$ and the development of lipid tubular structures is pivotal for the development of hierarchical cellular structures in general and plant cells in particular. Indeed, today's plant cells use tubular structures (up to hundreds of $\mathrm{nm}$ in diameter), called plasmodesmata, to connect neighboring cells across the cell wall in higher plant cells ${ }^{12}$. The plasmodesmata, which are connected to the endoplasmic reticulum (ER) in the interior of plant cells, are vital for intercellular communication, development and defense against pathogens ${ }^{8,12,34}$. These plasmodesmata are much 


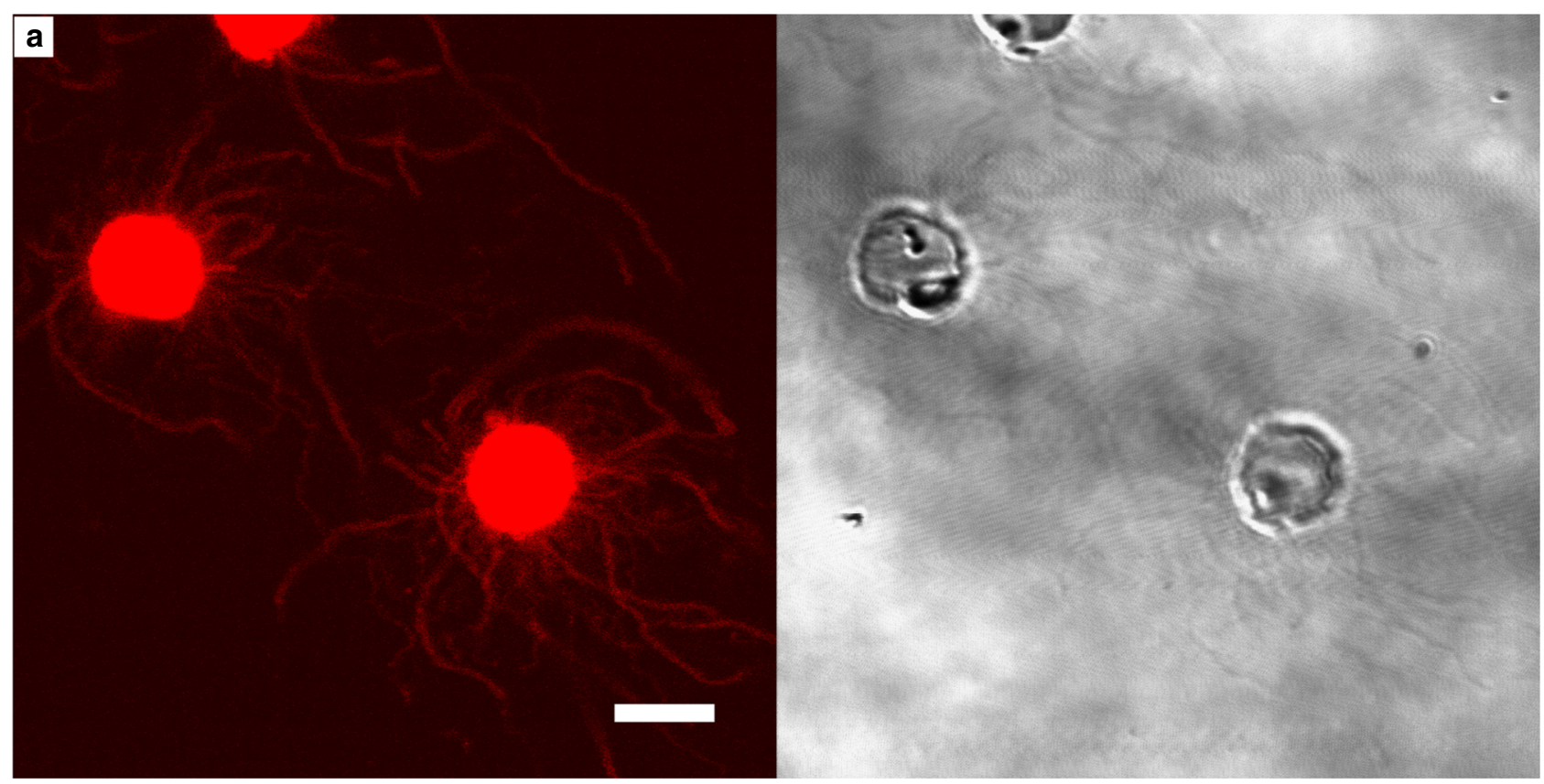

b
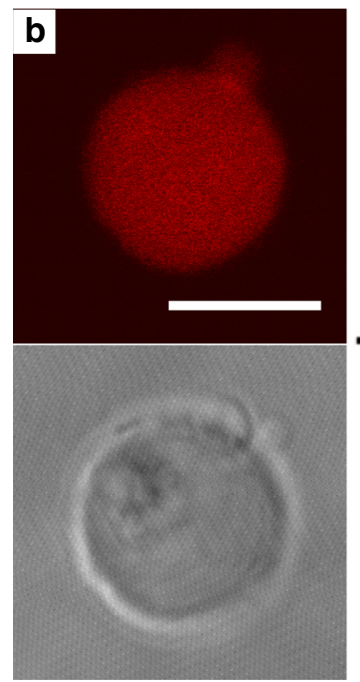
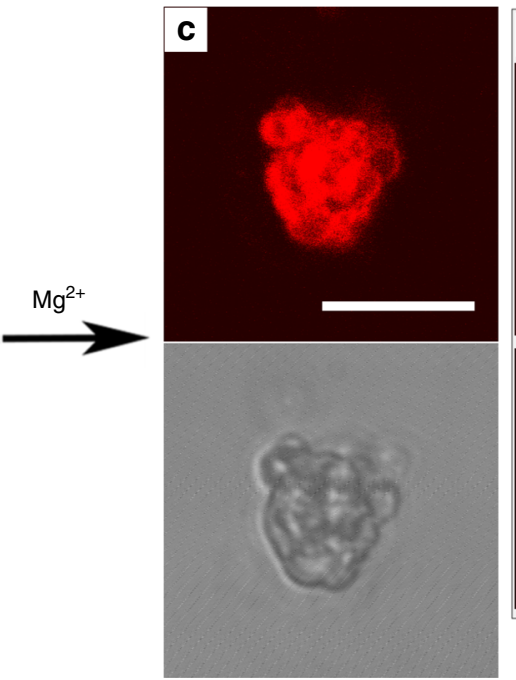

\section{d}
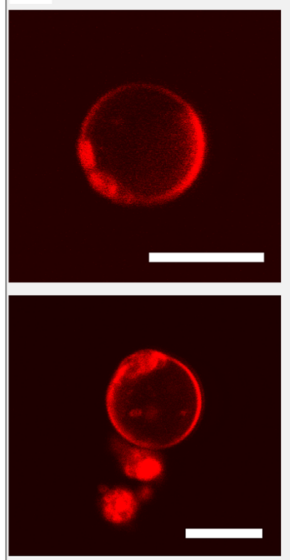

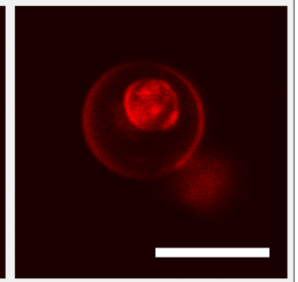

e

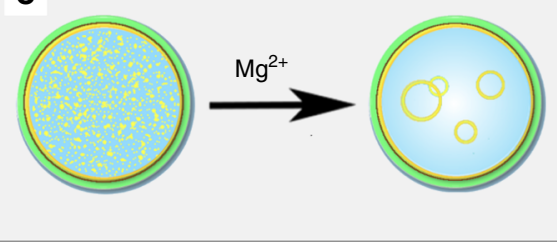

Fig. $\mathbf{6}$ Lipid tubular structure formation and expanded plantosomes after exposure to $\mathbf{M g}^{\mathbf{2}+}$ ions. CLSM images (fluorescence and transmission images) of a lipid tubular protrusions from expanded plantosomes. Lipid tubular protrusions were observed in $\sim 80 \%$ of the expanded plantosomes $(n=102$, obtained from eight experiments). Several, but not all, expanded plantosomes collapsed in the presence of $\mathrm{Mg}^{2+}: \mathbf{b}$ an expanded plantosome at pH 8.6 and c after exposure to $\mathrm{Mg}^{2+}$ (image taken at $10 \mathrm{mM}$ ). In some instances, the interior lipids were rearranged: $\mathbf{d}$ plantosomes with (occasional) giant vesicles in the interior (at $10 \mathrm{mM} \mathrm{Mg}^{2+}$ ). Images in $\mathbf{b}-\mathbf{d}$ are representative of two experiments. e Schematic representation of the fusion of small vesicles into larger vesicles and a continuous bilayer(s) at the plantosome inner interface that occurred for structures presented in d. Scale bars: $10 \mu \mathrm{m}$.

more complex than the present lipid tubular structures ${ }^{35}$. However, model systems are the main tool used to study membrane properties and in particular membrane tubulation ${ }^{36}$. Even though the expanded plantosomes reported here are simple, they open up possibilities for deriving, and studying the structure and properties of highly curved lipid membranes in a plant cell model system.

Divalent ions, such as $\mathrm{Mg}^{2+}$, are known to bridge and fuse small vesicles into larger vesicles ${ }^{16}$. In an attempt to reduce the internal pressure in the expanded plantosomes, we exposed them to magnesium ions. The concentration of $\mathrm{Mg}^{2+}$ was increased in a step-wise manner, 2, 5, and $10 \mathrm{mM}$. In most cases, the expanded plantosome structure collapsed (Fig. 6b, c) sometimes already in the presence of $2 \mathrm{mM} \mathrm{Mg}^{2+}$ (Supplementary Movie 4). However, in some cases, the interior vesicles were transformed into a continuous bilayer(s) at the plantosome inner interface, in a similar manner as observed for other systems ${ }^{16}$. These plantosomes could then withstand higher $\mathrm{Mg}^{2+}$ concentration up to $5 \mathrm{mM}$ (Supplementary Movie 4) or $10 \mathrm{mM}$, (Fig. 6d). In all of the cases, the tubular protrusions disappeared, suggesting that divalent ions changed the lipid packing in the plantosome membrane. Some interior vesicles also merged into giant vesicles that remained freely moving in the interior of the water-filled cavity (Fig, 6d, Supplementary Movie 4, schematic representation in Fig. 6e).

In conclusion, a strategy has been developed that overcomes the fundamental difficulties associated with assembling lipid vesicles with CNFs, which represents an important step toward advanced synthetic plant cells. The approach brings together different plant mimicking features and integrates lipid-mediated 
internalized structuration, expansion, and lipid tubular structure growth in the same microcapsule structures. Just like in natural parenchyma cells, our studies show the pivotal role that the CNF network has on restraining the expansion power of the interior aqueous environment. With our model, we show that a primitive fatty-acid-based membrane indeed can form tubular structures that stretch across a plant cell wall and we hypothesize that the cage-like plant cell wall and turgor pressure might have played an important role in the tubulation formation in primitive plant cells. The development of lipid tubular structures is a critical aspect of cell communication and the evolution of more hierarchical plant structures. The presented fabrication method opens up for future studies on more advanced plant cell models, permeability properties through plant cell wall/plasma membrane and intercellular ER models in different physiologically relevant settings and greater understanding of the evolution of plant cells. Additionally, this basic model could be further improved by increasing the complexity to answer the questions related to plasmolysis, ion responsiveness, and $\mathrm{pH}$ dependence of cell expansion. Finally, the fabrication protocol of plantosomes is useful, not only for model plant cells, but also in the production of model systems for algae, yeast, and bacteria.

\section{Methods \\ Materials. Cellulose nanofibers (CNFs) modified with cationic quaternary} ammonium groups ( $1.17 \mathrm{mmol} \mathrm{g}^{-1}$ fiber) was a kind gift (KTH, WWSC, Sweden). The CNFs were derived from never-dried softwood pulp (Nordic Paper Seffle AB, Sweden) and the reaction with glycidyltrimethylammonium chloride and homogenization steps were performed as described in detail earlier ${ }^{37}$. The CNFs have a high aspect ratio: the height is $2.5 \pm 0.8 \mathrm{~nm}$, obtained from atomic force microscopy (AFM) height measurements, and length is in micrometer range (Fig. 1a). The sugar beet pectin is a high methyl ester and naturally acetylated pectin $\left(\mathrm{Genu}^{\circledR}{ }^{\mathrm{BETA}}\right.$ pectin) extracted from sugar beet pulp. It was a kind gift from CP Kelco (Lille Skensved, Denmark). The pectin has a degree of esterification $\sim 55 \%$ and a degree of acetylation above $20 \%$. OA (purity $\geq 99 \%$ ), sodium chloride, chloroform (HiPerSolv $\geq 99.8 \%$ ), Rh-6G, SR-101, calcofluor-white stain stock solution (contains calcofluorwhite $\mathrm{M} 2 \mathrm{R}, 1 \mathrm{mg} \mathrm{mL}^{-1}$ and Evans blue $0.5 \mathrm{mg} \mathrm{mL}^{-1}$ ), sodium hydroxide, hydrochloric acid, POPC, POPE, Rh-DOPE, FITC-dextran (4 kDa, FITC:glucose = 1:250), were all purchased from Sigma-Aldrich (Sweden). Ammonium acetate (97\%) was purchased from Alfa Aesar GmbH (Germany) and ammonia solution (25\%) was purchased from Merck. $\mu$-Slides (four well) was purchased from Ibidi $\mathrm{GmbH}$ (Germany). All chemicals were used without further purification.

Preparation of working and stock solutions. All working solutions were prepared just prior to the experiments. A suspension of CNF (0.059 wt\%) in $100 \mathrm{mM} \mathrm{NaCl}$ was prepared by diluting a concentrated CNF suspension $(0.39 \mathrm{wt} \%)$ with MilliQwater and adding $\mathrm{NaCl}$ to obtain $100 \mathrm{mM} \mathrm{NaCl}$. Afterward, the CNF suspension was magnetically stirred overnight $(350 \mathrm{rpm})$ at room temperature (RT). The $\mathrm{pH}$ of the final suspension was $7.0 \pm 0.2$. To prepare $50 \mathrm{~mL}$ of the $0.2 \mathrm{wt} \%$ sugar beet pectin solution, $106 \mathrm{mg}$ of pectin was dissolved in MilliQ water in the presence of ca. $180 \mu \mathrm{l}$ of $1 \mathrm{M} \mathrm{NaOH}$ solution and magnetically stirred overnight at RT. The $\mathrm{pH}$ of the obtained solution was ca. 6.8. A $288 \mathrm{mM}$ OA in chloroform solution was prepared by mixing $0.94 \mathrm{~g}$ of OA with $15.64 \mathrm{~g}$ chloroform. The solution was stored at $4{ }^{\circ} \mathrm{C}$ prior to use. The POPC/POPE phospholipid stock solution was prepared by mixing POPC and POPE in a molar ratio of 5:1 in chloroform. The final concentration was $1 \mathrm{mM}$ POPC and $0.2 \mathrm{mM}$ POPE in chloroform and it was stored at $-20^{\circ} \mathrm{C}$. The Rh-DOPE/POPC/POPE phospholipid stock solution was prepared by mixing the fluorescently labeled phospholipid (Rh-DOPE) with POPC and POPE, the final concentration was $1 \mathrm{mM}$ POPC, $0.14 \mathrm{mM}$ POPE, and $0.09 \mathrm{mM}$ Rh-DOPE. The stock solution was stored at $-20^{\circ} \mathrm{C}$. Prior to the experiments all stock solutions were brought to RT.

Preparation of microcapsules. From the working solutions, $1.5 \mathrm{~g}$ of CNF suspension $(0.059 \mathrm{wt} \% \mathrm{CNF}$ in $100 \mathrm{mM} \mathrm{NaCl}, \mathrm{pH} 7 \pm 0.2)$ and $2.23 \mathrm{~g}$ of $\mathrm{OA}$ in chloroform $(288 \mathrm{mM})$ was added to a $15 \mathrm{~mL}$ Falcon tube (Supplementary Fig. 1a). The CNF-stabilized lipid droplets were obtained by mixing with an IKA T25 digital Ultra Turrax (24,000 rpm), Supplementary Fig. 1b. The mixing was carried out in a sequential way, i.e., $15 \mathrm{~s}$ mixing and $10 \mathrm{~s}$ pause, repeated three times, to ensure extensive mixing. After that, the droplets were allowed to settle for $\sim 15$ min prior to use. They phase separated into the upper water phase, middle CNF-stabilized oil droplets and lower chloroform phase, Supplementary Fig. 1c. The droplets, obtained from the middle part of the Falcon tube in Supplementary Fig. 1c, were immediately utilized to prepare microcapsules.
Equal amounts of the $0.2 \mathrm{wt} \%$ pectin working solution and a $200 \mathrm{mM} \mathrm{NaCl}$ in MilliQ-water solution ( $10 \mathrm{~g}$ each) were mixed (in a $40 \mathrm{~mL}$ glass vial) and the $\mathrm{pH}$ was adjusted to 10.0 with a $1 \mathrm{M}$ or $0.1 \mathrm{M} \mathrm{NaOH}$ solution. The final working concentration of the pectin solution was $0.1 \mathrm{wt} \%$ in $100 \mathrm{mM} \mathrm{NaCl}$. An amount of $300 \mu \mathrm{L}$ of the CNF-stabilized OA/chloroform droplets (middle phase, Supplementary Fig. 1c) was carefully taken and transferred into the pectin in 100 $\mathrm{mM} \mathrm{NaCl}$ solution and the chloroform was evaporated under magnetic stirring $(350 \mathrm{rpm})$ overnight $(15-18 \mathrm{~h})$. Though the initial $\mathrm{pH}$ of the pectin was 10.0, immediately after adding the CNF-stabilized oil droplets to the pectin solution, the $\mathrm{pH}$ of the dispersion was continuously dropping down during evaporation and stabilized at around $\mathrm{pH}$ 6.1. This is a consequence of the dissociation of OA into oleate in the presence of salt and/or higher $\mathrm{pHs}^{20}$. After evaporation, the microcapsules float to the surface of the solution due to the entrapped OA/oleate Then, the $\mathrm{pH}$ of the solution was adjusted to 2.0 with $100 \mathrm{mM} \mathrm{HCl}$, and after 30 min, the $\mathrm{pH}$ was raised to 6.5 with $100 \mathrm{mM} \mathrm{NaOH}$ to obtain the final microcapsules. Supplementary Fig. $3 c$, d shows the overall transformation of CNFstabilized lipid droplets into microcapsules, as derived from the microscopy images in Supplementary Fig. 3a. The microcapsule suspensions were stored at RT prior to further characterization.

Preparation of plantosomes. An amount of $0.5 \mathrm{~mL}$ of the POPC/POPE stock solution ( $1 \mathrm{mM} / 0.2 \mathrm{mM}$ POPC/POPE), $0.5 \mathrm{~mL}$ of chloroform, $1 \mathrm{~mL}$ of OA (288 $\mathrm{mM}$ stock solution), and $2.3 \mathrm{~g}$ of the CNF suspension ( $0.059 \mathrm{wt} \% \mathrm{CNF}$ in $100 \mathrm{mM}$ $\mathrm{NaCl}, \mathrm{pH} 7 \pm 0.2$ ) was added into a $15 \mathrm{~mL}$ Falcon tube and mixed an IKA T25 digital Ultra Turrax $(24,000 \mathrm{rpm}, 15 \mathrm{~s}$ mixing, $10 \mathrm{~s}$ pause, three repetitions). When the droplets also contained Rh-DOPE, $40 \mu \mathrm{L}$ of the Rh-DOPE/POPC/POPE phospholipid stock solution was included in the above mixture and $0.48 \mathrm{~mL}$ of the POPC/POPE stock solution was used instead of $0.5 \mathrm{~mL}$. The droplets were allowed to settle for $\sim 15 \mathrm{~min}$ prior to use, see Supplementary Fig. 1f. A light microscopy image of the obtained droplet is found in Fig. 3a, which shows that some of them contained very large water cavities in the interior. Droplets, obtained from the middle part of the Falcon tube, were collected.

These CNF-stabilized OA/phospholipid/chloroform droplets (taken from the middle phase, see Supplementary Fig. 1f) could not be directly transferred to a 0.1 wt $\%$ pectin in $100 \mathrm{mM} \mathrm{NaCl}$ solution ( $\mathrm{pH}$ 6.3), because aggregates were observed. To circumvent this, $300 \mu \mathrm{L}$ of the CNF-stabilized OA/phospholipid/chloroform droplets was first dispersed in $1.5 \mathrm{~mL}$ Eppendorf tube containing $0.7 \mathrm{~g}$ of the 200 $\mathrm{mM} \mathrm{NaCl}$ in MilliQ-water solution. The droplets were dispersed by repeatedly (trice) sucking and releasing the suspension with a Pasteur pipette, followed by transferring the dispersion to $9.3 \mathrm{~g}$ of $200 \mathrm{mM} \mathrm{NaCl}$ in MilliQ-water solution. Afterward, the suspension was transferred to the $0.2 \mathrm{wt} \%$ pectin solution $(10 \mathrm{~g}, \mathrm{pH}$ 6.8) present in a $40 \mathrm{~mL}$ glass vial. Now the droplets were in a $0.1 \mathrm{wt} \%$ pectin in 100 $\mathrm{mM} \mathrm{NaCl}$ solution with $\mathrm{pH}$ 6.3. The chloroform was evaporated under magnetic stirring $(350 \mathrm{rpm})$ overnight $(15-18 \mathrm{~h})$. The $\mathrm{pH}$ of the solution dropped (due to deprotonation of $\mathrm{OA}$ ) during evaporation and stabilized at around $\mathrm{pH}$ 5.8-5.9. The tubes and glass vials were protected from light using aluminum foil when RhDOPE was present. The final suspension was a mixture of capsules with large water-filled cavities (plantosomes), and in some cases, the water cavity was missing (microcapsules), see Fig. 3b. The plantosomes yield was $44 \pm 21 \%$ (calculated from the number of plantosomes prior to and after chloroform evaporation). The suspensions were stored at RT prior to further experiments.

Light microscopy. Microcapsules and plantosomes, both during preparation and final structures (Fig. 1e, f, Fig. 3a, b, Supplementary Figs. 3a and 5), were studied with upright light microscope (VisiScope, VWR, Sweden) equipped with VisiCam 16 Plus camera (IS VisiCam Image Analyser 3.9.0.605 software), and 40x and 20x air objectives. Plantosomes were also studied using an Axio Vert.A1 Light Microscope (Carl Zeiss, Germany) equipped with a Zeiss AxioCam 305 color camera (Zeiss Zen 2.6 (blue edition) software), and $20 \times$ and $40 \times$ air objectives. The size of microcapsules and plantosomes, at different conditions, were measured with Image J 1.50b (NIH, USA) or Zeiss Zen 2.6 (blue edition).

To calculate the plantosome yield, $100 \mu \mathrm{L}$ of suspension was dropped on a microscopic slide and covered with a coverslip and the number of plantosomes in the suspension were calculated from 20 images $(151 \geq n \geq 35)$, which were randomly taken ( $20 \times$ objective, Axio Vert.A1 Light Microscope, Zeiss Zen 2.6 (blue edition)). The yield was calculated from the number of plantosomes prior to and after chloroform evaporation. The yield is an average from five separate experiments (yields $26 \%, 38 \%, 30 \%, 45 \%$, and $80 \%$ ).

Polarized optical microscopy. The organization of lipids in the interior of the microcapsules, plantosomes, and expanded plantosomes was studied using an inverted Axio Vert.A1 Light Microscope (Carl Zeiss, Germany, Zeiss Zen 2.6 (blue edition) software) equipped with cross-polarized light filters and with $10 \times, 20 \times$, and $40 \times$ air objectives and a Zeiss AxioCam 305 color camera.

$\mathrm{POM}$ was used to monitor the change of molecular organization of OA/oleate inside microcapsules (microcapsule with only OA/oleate cores) in acidic and alkaline conditions, as well as the lipid release from microcapsule interior and microcapsule wall expansion. The results presented in Supplementary Movie 1. A $100 \mu \mathrm{L}$ microcapsule suspension, containing crumpled microcapsule structures 
obtained after chloroform evaporation, see light microscopy image in Supplementary Fig. 3a, after evaporation, was dropped on a microscopic slide and covered with a coverslip. The experimental setup is shown in Supplementary Fig. 12. A $100 \mathrm{mM} \mathrm{HCl}$ solution was added at the rate of $2 \mu \mathrm{L} \mathrm{min}^{-1}$ using a syringe pump (New Era Pump systems, USA), until the microcapsule appeared spherical in shape. Afterward, a $100 \mathrm{mM} \mathrm{NaOH}$ was added at the same rate to observe lipid release and microcapsule expansion. The empty microcapsule walls were stained with calcofluor-white stain stock solution $\left(1.5 \mathrm{mg} \mathrm{mL}^{-1}\right)$, with the same pumping rate. After the dye reached the observation point, the flow was stopped and different capsules were imaged.

Atomic force microscopy. CNF (0.05 wt\%) was dispersed in water and stirred overnight (350 rpm) at RT then the CNF was sonicated with Sonics Vibra-Cell, $80 \%$ amplitude, $750 \mathrm{~W}, 1 / 2^{\prime \prime}$ tip for $60 \mathrm{~s}$, ( $30 \mathrm{~s}$ on, $10 \mathrm{~s}$ off). Then the CNF was further diluted with water and $0.0025 \mathrm{wt} \% \mathrm{CNF}$ was obtained. Plasma activated clean silicon wafer was dipped $(1 \mathrm{~min})$ in the CNF suspension and dried with nitrogen and AFM images were obtained (Scanasyst-air cantilever) using Bruker NanoScope V (U.S.A.). Images of $2 \times 2 \mu \mathrm{m}$ in size with $512 \times 512$-pixel resolution were recorded using the software NanoScope 8.15 . The images were analyzed using Gwyddion 2.47 .

Quartz crystal microbalance with dissipation. The interaction of CNF and pectin was examined by Quartz crystal microbalance with dissipation (QCM-D), model E4, (Q-Sense, Sweden) using Au-coated quartz crystals. Before the experiments, the crystals were thoroughly cleaned with the procedure described earlier ${ }^{38}$ and the adsorption steps were carried out at $25^{\circ} \mathrm{C}$ with a constant flow rate of $100 \mu \mathrm{L} \mathrm{min}^{-1}$. The QCM-D results for CNF $(0.059 \mathrm{wt} \%$ in $100 \mathrm{mM} \mathrm{NaCl}, \mathrm{pH} 7 \pm$ $0.2)$ and pectin ( $0.1 \mathrm{wt} \%$ pectin in $100 \mathrm{mM} \mathrm{NaCl}, \mathrm{pH} 6.3)$ interaction and only the adsorption of pectin on the Au-sensor is shown in Supplementary Fig. 4c. Prior to and after the adsorption of CNF or pectin, a washing step was included using $100 \mathrm{mM} \mathrm{NaCl}$ in MilliQ-water. Sufficient time was allowed until a steady QCM-D signal was attained for each step.

Cryo-transmission electron microscopy. Samples were imaged with a JEM-2100f (Jeol Ltd., Japan). A drop of the suspension containing vesicles was added to a Quantifoil holey carbon grid (R2/2, 200 mesh) and plunge-frozen (FEI Vitrobot Mark III). The vesicle suspension was prepared almost in the same way as plantosomes are prepared, but in the absence of CNF and pectin. A droplet of the lipid in chloroform (consisting of $173 \mu \mathrm{L}$ of $288 \mathrm{mM}$ OA stock solution, $83 \mu \mathrm{L}$ of POPC/ POPE stock solution, and $6.92 \mu \mathrm{L}$ of the Rh-DOPE/POPC/POPE phospholipid stock solution) was added to $20 \mathrm{~g}$ of a $100 \mathrm{mM} \mathrm{NaCl}$ solution in MilliQ-water (pH ca. 6). The solution was put on magnetic stirring $(350 \mathrm{rpm})$ overnight $(17 \mathrm{~h})$ to allow the chloroform to evaporate. The following day, $20 \mathrm{~g}$ of a solution consisting

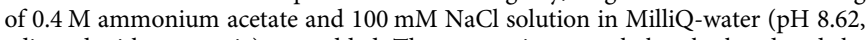
adjusted with ammonia) was added. The suspension was shaken by hand and the $\mathrm{pH}$ was adjusted to 8.6 with ammonia. Afterward, the suspension was passed six times through a $0.2 \mu \mathrm{m}$ syringe filter. The lipid concentration in the final suspension was $1.2 \mathrm{mM}$.

Transmission electron microscopy. High-magnification transmission electron micrographs of the microcapsules/plantosomes were acquired using a TEM from Hitachi, model HT7700 (Japan, Hitachi HT7700 02.05 software) at an accelerating voltage of $100 \mathrm{kV}$ in high-contrast mode. The microcapsules/plantosomes (after chloroform evaporation) were first washed with water (suspension diluted 20 times with MilliQ-water, separation of microcapsules/plantosomes from water phase. Taking $200 \mu \mathrm{L}$ of the separated microcapsules/plantosomes and diluting with 800 $\mu \mathrm{L}$ MilliQ) and deposited onto 200 mesh Formvar/carbon TEM grids (Ted Pella, $01800-\mathrm{F}$ ) and thoroughly air-dried. Then the lipid contents were removed by dipping the TEM grid into $2.5 \mathrm{~mL}$ of $100 \mathrm{mM} \mathrm{NaOH}$ solution for 3 min followed by thorough washing with MilliQ water (dipping into $2.5 \mathrm{~mL}$ MilliQ-water for $2 \mathrm{~min}$ and repeating a second time with fresh MilliQ-water) and air-dried before imaging. The images were taken without staining.

Scanning electron microscopy. After TEM analysis, the same samples (on TEM grids) were grounded with $\mathrm{Pt} / \mathrm{Pd}(60 / 40)$ for $20 \mathrm{~s}$ at a current of $80 \mathrm{~mA}$ using a Cressington 208HR sputter coater and high-resolution scanning electron micrographs were acquired using a SEM from Hitachi, model S-4800 (Japan, S- 4800 04.05 software) at an accelerating voltage of $1 \mathrm{kV}$ (Supplementary Fig. 6a, b).

Confocal laser scanning microscopy. CLSM imaging was performed using a Zeiss $780 \mathrm{UV} /$ Vis (Zeiss, Germany, Zen Black 2012 software) equipped with CApichromat $40 \times / 1.2 \mathrm{NA}$ water immersion objectives at RT (Figs. $2 \mathrm{a}-\mathrm{d}, 3 \mathrm{~d}-\mathrm{f}$ and $4 \mathrm{a}, \mathrm{b}, \mathrm{f}$ in the main manuscript) or a LSM 510 UV/Vis (Zeiss, Germany, LSM 510 $3.2 \mathrm{SP} 2$ software) equipped with $40 \times / 1.3 \mathrm{NA}$ air objectives (Figs. 5 and 6 , Supplementary Figs. 8, 9 and 11). Just prior to the experiments, the fluorophores Rh-6G (lipophilic dye ${ }^{23}$, see Supplementary Note 8), SR-101 (hydrophilic dye), and $4 \mathrm{kDa}$ FITC-dextran with concentrations $0.02,1.0$, and $2 \mathrm{mg} \mathrm{mL}^{-1}$, respectively, were prepared in $100 \mathrm{mM} \mathrm{NaCl}$ and the $\mathrm{pH}$ was adjusted to 6.5 (Figs. 2, 3 and $4 \mathrm{f}$ ). The outer encasing CNF-rich plantosome wall was revealed by exposing the plantosomes to cellulose-specific calcofluor-white stain (stock solution $1.5 \mathrm{mg} \mathrm{mL}^{-1}$ ). An equal volume of the freshly prepared fluorophores and the microcapsule (or plantosome) suspension were mixed and the microcapsules were studied with CLSM.

The permeability of $4 \mathrm{kDa}$ FITC-dextran through expanded plantosomes (that also contained Rh-DOPE, results in Supplementary Fig. 9) was studied using $4 \mathrm{kDa}$ FITC-dextran (stokes radius of $\sim 1.4 \mathrm{~nm}$, producer's information) at a concentration of $1 \mathrm{mg} \mathrm{mL}^{-1}$ prepared in $0.2 \mathrm{M}$ ammonium acetate, $100 \mathrm{mM} \mathrm{NaCl}$, pH 8.8 or 8.7 (adjusted with ammonia). Details of the experimental setup used to expand and study the plantosomes are given in the next section. The laser excitation wavelengths used were $514 \mathrm{~nm}$ (Rh-6G), $561 \mathrm{~nm}$ (SR-101), $488 \mathrm{~nm}$ (FITC-dextran), 561 or $543 \mathrm{~nm}$ (Rh-DOPE), and $405 \mathrm{~nm}$ (calcofluor-white). Images were analyzed with Zeiss Zen 2.6 (blue edition) or Zeiss LSM Image Browser.

Experimental setup for in situ monitoring with CLSM. The effect of different $\mathrm{pH}$ values and $\mathrm{MgCl}_{2}$ concentrations was studied (in the presence of ammonium acetate and $\mathrm{NaCl}$ ) by using the plantosomes/microcapsule/expanded plantosomes with Rh-DOPE and CLSM. Details of the CLSM instruments and objectives used are found in the previous section. To study the effect of $\mathrm{pH}, 0.2 \mathrm{M}$ ammonium acetate was prepared in $0.1 \mathrm{M} \mathrm{NaCl}$ solution and the $\mathrm{pH}$ was adjusted to $6.5,8.2$, 8.4, and 8.7-8.8 (with ammonia). A $0.4 \mathrm{M}$ ammonium acetate in $0.1 \mathrm{M} \mathrm{NaCl}$ solution, $\mathrm{pH}$ 6.5, was also prepared. Different concentrations of $\mathrm{MgCl}_{2}(2,5$, and $10 \mathrm{mM}$ ) were prepared in a solution composed of $0.1 \mathrm{M} \mathrm{NaCl}$ and $0.2 \mathrm{M}$ ammonium acetate, and the $\mathrm{pH}$ was adjusted to 8.7 or 8.8 (with ammonia). In situ observations were performed using a modified $\mu$-slide well (Ibidi, Germany), as shown in Supplementary Fig. 13. The spacer, cover-slide, and tubes were glued using thiol resin. First $100 \mu \mathrm{L}$ of capsule suspension (plantosomes and microcapsules) was added, and plantosomes/capsules entered the space between the cover slides (space ca. $400 \mu \mathrm{m}$ in height) due to capillary forces. Then $500 \mu \mathrm{L}$ of a $0.1 \mathrm{M} \mathrm{NaCl}$ solution in MilliQ water was added. The setup was placed in the microscope and the tubes were connected to pumps and the area of interest was located. Then $500 \mu \mathrm{L}$ of $0.2 \mathrm{M}$ ammonium acetate solution (pH 6.5) followed by $500 \mu \mathrm{L}$ of $0.4 \mathrm{M}$ ammonium acetate solution ( $\mathrm{pH} 6.5$ ) was added carefully with the pipette and equilibrated for $\sim 1 \mathrm{~h}$. Now the surrounding medium was a $0.19 \mathrm{M}$ ammonium acetate, $0.1 \mathrm{M} \mathrm{NaCl}, \mathrm{pH} \sim 6.5$. After that the $0.2 \mathrm{M}$ ammonium acetate solutions with different $\mathrm{pH}(8.2,8.4$, and 8.7 or 8.8$)$ were pumped (using syringe pump) one by one at the rate of $200 \mu \mathrm{L} \mathrm{min}^{-1}$ and the solutions was pumped out (using a peristaltic pump) from the chamber at the same speed and collected (Supplementary Fig. 13). For the collected liquid $(1.5 \mathrm{~mL}$ aliquot) at the outlet, the $\mathrm{pH}$ was recorded and reported. During the final $\mathrm{pH}$ increase (from 8.3 to 8.6 ), the targeted $\mathrm{pH}$ was set to $8.60-8.69$, and either a solution with $\mathrm{pH} 8.7$ or 8.8 was used to reach this final pH. Results are found in Figs. 5 and 6a, Supplementary Movie 2 and 3, Supplementary Figs. 8, 9 and 11. After that, $\mathrm{MgCl}_{2}$ solutions (2, 5, and $10 \mathrm{mM}$ ) was pumped $\left(200 \mu \mathrm{L} \mathrm{min}^{-1}\right)$ and the changes were recorded (results in Fig. $6 \mathrm{~b}-\mathrm{d}$ and Supplementary Movie 4). Here, the $\mathrm{pH}$ was also set to a $\mathrm{pH}$ between 8.60 and 8.69 , and to achieve this, $\mathrm{MgCl}_{2}$ solutions (composition described above) with either $\mathrm{pH} 8.7$ or 8.8 was used. Each solution was pumped for $45-60 \mathrm{~min}$.

Reporting summary. Further information on research design is available in the Nature Research Reporting Summary linked to this article.

\section{Data availability}

Data supporting the main findings of this work are available within the paper and its Supplementary Information files. A reporting summary for this Article is available as a Supplementary Information file. Additional data generated and analyzed during the current study are available from the corresponding author upon request. The source data underlying Fig. $5 \mathrm{k}, \mathrm{l}$ are provided as a Source Data file.

Received: 15 August 2019; Accepted: 30 January 2020; Published online: 19 February 2020

\section{References}

1. Villar, G., Graham, A. D. \& Bayley, H. A tissue-like printed material. Science 340, 48-52 (2013).

2. Luisi, P. L. The Emergence of Life: from Chemical Origins to Synthetic Biology (Cambridge University Press, 2006).

3. Kumar, B. V. V. S. P., Patil, A. J. \& Mann, S. Enzyme-powered motility in buoyant organoclay/DNA protocells. Nature Chemistry 10, 1154-1163 2018).

4. Joesaar, A. et al. DNA-based communication in populations of synthetic protocells. Nat. Nanotechnol. 14, 369-378 (2019).

5. Stano, P. Is research on "synthetic cells" moving to the next level? Life 9, 3 (2018).

6. Xu, C., Hu, S. \& Chen, X. Artificial cells: from basic science to applications. Mater. Today 19, 516-532 (2016). 
7. Gopfrich, K., Platzman, I. \& Spatz, J. P. Mastering complexity: towards bottom-up construction of multifunctional eukaryotic synthetic cells. Trends Biotechnol. 36, 938-951 (2018).

8. Lucas, W. J. et al. Selective trafficking of KNOTTED1 homeodomain protein and its mRNA through plasmodesmata. Science 270, 1980-1983 (1995).

9. Grison, M. S. et al. Specific membrane lipid composition is important for plasmodesmata function in Arabidopsis. Plant Cell 27, 1228-1250 (2015)

10. Brunkard, J. O., Runkel, A. M. \& Zambryski, P. C. The cytosol must flow: intercellular transport through plasmodesmata. Curr. Opin. Cell Biol. 35, 13-20 (2015).

11. Navon, Y., Radavidson, H., Putaux, J.-L., Jean, B. \& Heux, L. pH-sensitive interactions between cellulose nanocrystals and DOPC liposomes. Biomacromolecules 18, 2918-2927 (2017).

12. Buchanan, B. B., Gruissem, W. \& Jones, R. L. Biochemistry \& Molecular Biology of Plants. (American Society of Plant Physiologists, 2000).

13. Cosgrove, D. J. Growth of the plant cell wall. Nat. Rev. Mol. Cell Biol. 6, 850 (2005).

14. Svagan, A. J., Samir, M. A. S. A. \& Berglund, L. A. Biomimetic polysaccharide nanocomposites of high cellulose content and high toughness. Biomacromolecules 8, 2556-2563 (2007).

15. Svagan, A. J. et al. Cellulose nanofiber/nanocrystal reinforced capsules: a fast and facile approach toward assembly of liquid-core capsules with high mechanical stability. Biomacromolecules 15, 1852-1859 (2014).

16. Weiss, M. et al. Sequential bottom-up assembly of mechanically stabilized synthetic cells by microfluidics. Nat. Mater. 17, 89 (2017).

17. Kalashnikova, I., Bizot, H., Cathala, B. \& Capron, I. New pickering emulsions stabilized by bacterial cellulose nanocrystals. Langmuir 27, 7471-7479 (2011).

18. Hanczyc, M. M., Fujikawa, S. M. \& Szostak, J. W. Experimental models of primitive cellular compartments: encapsulation, growth, and division. Science 302, 618-622 (2003).

19. Mansy, S. S. et al. Template-directed synthesis of a genetic polymer in a model protocell. Nature 454, 122-U110 (2008).

20. Mele, S. et al. Phase behavior in the biologically important oleic acid/sodium oleate/water system. Chem. Phys. Lipids 211, 30-36 (2018).

21. Suga, K., Kondo, D., Otsuka, Y., Okamoto, Y. \& Umakoshi, H. Characterization of aqueous oleic acid/oleate dispersions by fluorescent probes and raman spectroscopy. Langmuir 32, 7606-7612 (2016).

22. Edwards, K., Silvander, M. \& Karlsson, G. Aggregate structure in dilute aqueous dispersions of oleic acid/sodium oleate and oleic acid/sodium oleate/ egg phosphatidylcholine. Langmuir 11, 2429-2434 (1995).

23. Han, H. S., Song, J., Hong, J., Kim, D. \& Kang, T. Immiscible oil-water interface: dual function of electrokinetic concentration of charged molecules and optical detection with interfacially trapped dold nanorods. Anal. Chem. 86, 6160-6165 (2014).

24. Ohlrogge, J. \& Browse, J. Lipid biosynthesis. Plant Cell 7, 957-970 (1995).

25. Murphy, A. S., Peer, W. \& Schulz, B. The Plant Plasma Membrane. (Springer, 2011).

26. Zhang, T., Zheng, Y. Z. \& Cosgrove, D. J. Spatial organization of cellulose microfibrils and matrix polysaccharides in primary plant cell walls as imaged by multichannel atomic force microscopy. Plant J. 85, 179-192 (2016).

27. Carpita, N., Sabularse, D., Montezinos, D. \& Delmer, D. P. Determination of the pore size of cell walls of living plant cells. Science 205, 1144-1147 (1979).

28. Zhu, T. F. \& Szostak, J. W. Coupled growth and division of model protocell membranes. J. Am. Chem. Soc. 131, 5705-5713 (2009).

29. Parker, R. M. et al. Hierarchical self-assembly of cellulose nanocrystals in a confined geometry. ACS Nano 10, 8443-8449 (2016).

30. Svagan, A. J. et al. Liquid-core nanocellulose-shell capsules with tunable oxygen permeability. Carbohyd. Polym. 136, 292-299 (2016).

31. Hentrich, C. \& Szostak, J. W. Controlled growth of filamentous fatty acid vesicles under flow. Langmuir 30, 14916-14925 (2014).

32. Fujiwara, K. \& Yanagisawa, M. Liposomal internal viscosity affects the fate of membrane deformation induced by hypertonic treatment. Soft Matter 13 9192-9198 (2017).

33. Szostak, J. W., Bartel, D. P. \& Luisi, P. L. Synthesizing life. Nature 409, 387-390 (2001).
34. Bayer, E. M., Mongrand, S. \& Tilsner, J. Specialized membrane domains of plasmodesmata, plant intercellular nanopores. Front. Plant Sci. 5, 507 (2014).

35. Knox, K. et al. Putting the squeeze on Plasmodesmata: a role for reticulons in primary plasmodesmata formation. Plant Physiol. 168, 1563-1572 (2015).

36. Pomorski, T. G., Nylander, T. \& Cárdenas, M. Model cell membranes: discerning lipid and protein contributions in shaping the cell. Adv. Colloid Interface Sci. 205, 207-220 (2014).

37. Pei, A., Butchosa, N., Berglund, L. A. \& Zhou, Q. Surface quaternized cellulose nanofibrils with high water absorbency and adsorption capacity for anionic dyes. Soft Matter 9, 2047-2055 (2013).

38. Paulraj, T. et al. Bioinspired layer-by-layer microcapsules based on cellulose nanofibers with switchable permeability. Biomacromolecules 18, 1401-1410 (2017).

\section{Acknowledgements}

T.P and A.J.S would like to acknowledge SSF (ICA14-0045) for financial support. Dr. P. Purhonen is acknowledged for help with TEM imaging. Prof. S. Mann is acknowledged for valuable feedback. Open access funding provided by Royal Institute of Technology.

\section{Author contributions}

T.P. performed and analyzed light microscopy, POM, QCM-D, AFM measurements, and designed the modified Ibidi well experiments. T.P. and S.W. performed and analyzed the CLSM measurements. D.C.F.W and A.D. improved the understanding of the lipid assembly. A.V.R. performed SEM and TEM imaging. A.J.S. designed the experiments and preparation protocol of the plantosomes/microcapsules. A.J.S, M.C., and T.G.P. outlined the manuscript, and all authors contributed to the writing of the manuscript.

\section{Competing interests}

The authors declare no competing interests.

\section{Additional information}

Supplementary information is available for this paper at https://doi.org/10.1038/s41467 020-14718-x.

Correspondence and requests for materials should be addressed to A.J.S.

Peer review information Nature Communications thanks Yohann Boutte, and the other, anonymous, reviewer(s) for their contribution to the peer review of this work

Reprints and permission information is available at http://www.nature.com/reprints

Publisher's note Springer Nature remains neutral with regard to jurisdictional claims in published maps and institutional affiliations.

Open Access This article is licensed under a Creative Commons Attribution 4.0 International License, which permits use, sharing, adaptation, distribution and reproduction in any medium or format, as long as you give appropriate credit to the original author(s) and the source, provide a link to the Creative Commons license, and indicate if changes were made. The images or other third party material in this article are included in the article's Creative Commons license, unless indicated otherwise in a credit line to the material. If material is not included in the article's Creative Commons license and your intended use is not permitted by statutory regulation or exceeds the permitted use, you will need to obtain permission directly from the copyright holder. To view a copy of this license, visit http://creativecommons.org/ licenses/by/4.0/

(c) The Author(s) 2020 